Bundesgesundheitsbl 2015 · 58:908-920

DOI 10.1007/s00103-015-2192-8

Online publiziert: 8. Juli 2015

c) Springer-Verlag Berlin Heidelberg 2015

CrossMark

Zum Schutz von Badenden vor Cyanobakterien-Toxinen hat das Umweltbundesamt im Bundesgesundheitsblatt Empfehlungen veröffentlicht, zuletzt in 2003 [1]. Ihre Aktualisierung ist nunmehr nicht nur aufgrund neuer wissenschaftlicher Erkenntnisse erforderlich, sondern auch aufgrund vielfältiger Praxiserfahrungen sowie des Vergleichs zu Regelungen in anderen Ländern [2]. Ferner fordert die EGBadegewässerrichtlinie [3] in ihrer aktuellen Fassung neben der Bewertung fäkaler Verunreinigungen auch die Erfassung des Vorkommens von Algen und Cyanobakterien (Blaualgen). Ziel der „Empfehlung zum Schutz von Badenden vor Cyanobakterien-Toxinen" ist nach wie vor unter Beachtung des vorsorgenden Gesundheitsschutzes eine Freizeit-Nutzung auch eutropher Gewässer zu ermöglichen. Wenn auch erfreulicherweise das Vorkommen von Cyanobakterien in einigen Badegewässern rückläufig ist [4], so sind sie dennoch weiterhin weit verbreitet und stellen vor allem bei Massenentwicklungen ein Gesundheitsrisiko in Badegewässern dar.

Das Umweltbundesamt veröffentlicht auf Empfehlung der Badewasserkommission des Umweltbundesamtes (BWK) und des Bund-Länder-Arbeitskreises Badegewässer (BLAK) die folgende Aktualisierung der Empfehlung zum Schutz von Badenden vor Cyanobakterien-Toxinen. Sie ist primär auf Binnen-Badegewässer zugeschnitten; unter Berücksichtigung der höheren räumlichen Variabilität des Cyanobakterien-Vorkommens in Küstengewässern können Teile dieser Empfehlung auch zu deren Beurteilung und Überwachung angewandt werden.

\section{Hintergrundinformationen}

\subsection{Cyanobakterien-Toxine}

Cyanobakterien können eine Vielzahl von Wirkstoffen bilden, mit sehr unterschied-

\title{
Empfehlung zum Schutz von Badenden vor Cyanobakterien-Toxinen
}

lichen und z. T. stark toxischen Wirkungen - auch "Cyanotoxine" genannt. In - Tab. 1 sind die wichtigsten heute bekannten Toxine von Süß- und Brackwasser-Cyanobakterien zusammengestellt. Es handelt sich dabei insbesondere um hepato- und neurotoxische Peptide und Alkaloide mit zum Teil auffällig hoher akuter Toxizität in tierexperimentellen Untersuchungen. Für viele weitere Substanzen aus Cyanobakterien ist ihre Wirkung erst in Ansätzen untersucht worden oder es steht noch eine Strukturaufklärung aus. Auf der Basis des aktuellen Kenntnisstandes wird angenommen, dass mit den in - Tab. 1 dargestellten Stoffen die wichtigsten systemisch wirkenden Cyanotoxine beschrieben sind, die Entdeckung weiterer Cyanotoxine kann jedoch nicht ausgeschlossen werden.

Manche Cyanotoxine (insbesondere Microcystine, Nodularine) sind konstitutionelle Zellbestandteile, d. h., sie werden immer synthetisiert, sofern die Cyanobakterien das Gen für ihre Bildung besitzen. Andere Toxine werden unter bestimmten Wachstumsbedingungen, wie z. B. Cylindrospermopsin bei höheren Temperaturen $\left(>25-30^{\circ} \mathrm{C}\right)$, nicht mehr synthetisiert. Einige Cyanotoxine wie Microcystine und Nodularine treten vorwiegend intrazellulär auf und werden kaum ausgeschieden. Größere Mengen an gelösten Microcystinen findet man deshalb nur nach massenhaftem Absterben von Zellen, wenn das Toxin durch deren Zersetzung frei wird. In der Regel führen Verdünnung mit dem umgebenden Wasser sowie mikrobieller Abbau mit Halbwertszeiten von wenigen Tagen zu einem schnellen Rückgang der gelöst im Wasser vorkommenden Microcystine. Dagegen werden andere Cyanotoxine wie Cylindrospermopsin oder Anatoxin-a auch von intakten Zellen zeitweise zu einem hohen Anteil in das die Zellen umgebende Wasser abgegeben. Da im Wasser gelöstes Cylindro- spermopsin manchmal kaum abgebaut wird, kann dieses Toxin auch noch Monate nach Zusammenbruch der produzierenden Organismen im Wasser nachgewiesen werden [4].

Für die Badegewässer in Deutschland sind aufgrund ihres häufigen Vorkommens in ggf. gesundheitlich relevanten Konzentrationen in Binnengewässern die Microcystine sowie an der Ostsee das strukturell ähnliche Nodularin von besonderer Bedeutung. Microcystine sind aus 7 verschiedenen Aminosäuren zusammengesetzte zyklische Heptapeptide mit einem Molekulargewicht im Bereich von ca. 900-1100 Da. Bisher wurden mehr als 90 verschiedene Strukturvarianten beschrieben, von denen insbesondere das sehr häufig vorkommende MicrocystinLR toxikologisch relativ gut untersucht ist, während Informationen über die anderen Strukturvarianten vorwiegend auf Daten zur akuten Toxizität (z. B. LD $_{50}$ i.p. Maus) beschränkt sind [5].

Akute Leberschädigungen und tödliche Vergiftungsfälle durch Microcystine (und Nodularine) sind für Haus- und Nutztiere sowie wild lebende Tiere (z. B. Fische und Vögel) vielfach beschrieben worden. Darüber hinaus zeigen verschiedene tierexperimentelle Studien dosisabhängig toxische Effekte, wie eine Gewichtsreduktion sowie funktionale und strukturelle Leberveränderungen (z. B. Erhöhung der Leberenzyme, Einblutungen, Gewebsnekrosen) [5, 6]. Auf molekularer Ebene hemmt Microcystin aufgrund einer kovalenten Bindung die Proteinphosphataseaktivität. Ferner beschreiben einige Untersuchungen Microcystine als Tumorpromotoren, und chinesische Untersuchungen zeigen für eine Bevölkerungsgruppe eine Korrelation zwischen dem Auftreten primärer Leberzellkarzinome und dem Vorkommen von Microcystinen in Rohwasser, das unmittelbar aus Oberflächenwasser gewonnen 
Tab. 1 Übersicht der bekannten Cyanotoxine, ihrer chemischen Struktur sowie des Kenntnisstandes zu ihrer Toxizität und Wirkungsweise [5-7]

\begin{tabular}{|c|c|c|c|c|c|c|}
\hline \multirow[t]{2}{*}{ Cyanotoxin } & \multicolumn{6}{|c|}{ Informationen verfügbar über } \\
\hline & Struktur & $\begin{array}{l}\text { Akute Toxizität } \\
\text { i.p. Maus }\left(\mathrm{LD}_{50} \text { in }\right. \\
\mu \mathrm{g} / \mathrm{kg} \text { Körperge- } \\
\text { wicht) }\end{array}$ & $\begin{array}{l}\text { Akute Toxizität } \\
\text { oral }\left(\operatorname{LD}_{50} \text { in } \mu \mathrm{g} /\right. \\
\text { kg Körperge- } \\
\text { wicht) }\end{array}$ & $\begin{array}{l}\text { Chronisch. Toxi- } \\
\text { zität oral (NOAEL } \\
\text { oder LOAEL } \mu \mathrm{g} / \mathrm{kg} \\
\text { Körpergewicht) }\end{array}$ & $\begin{array}{l}\text { Mechanismus der } \\
\text { Toxizität }\end{array}$ & Karzinogenität \\
\hline Microcystin-LR & $\begin{array}{l}\text { Zyklische } \\
\text { Peptide mit } \\
\text { ADDA }\end{array}$ & $60(25-125)$ & 5000 & $40-100$ & $\begin{array}{l}\text { Blockiert Protein-Phospha- } \\
\text { tasen } 1 \text { und } 2 \mathrm{~A} \text { insb. in der } \\
\text { Leber } \\
\rightarrow \text { Zerstörung des Leberge- } \\
\text { webes, inneres Verbluten }\end{array}$ & $\begin{array}{l}\text { IARC Gruppe 2B } \\
\text { („possibly carcino- } \\
\text { genic to humans") }\end{array}$ \\
\hline $\begin{array}{l}\text { Andere Microcys- } \\
\text { tine }\end{array}$ & $\begin{array}{l}\text { Zyklische } \\
\text { Peptide mit } \\
\text { ADDA }\end{array}$ & $60->1200$ & Keine Daten & Keine Daten & $\begin{array}{l}\text { Blockiert Protein-Phospha- } \\
\text { tasen } 1 \text { und } 2 A \text { insb. in der } \\
\text { Leber } \\
\rightarrow \text { Zerstörung des Leberge- } \\
\text { webes, inneres Verbluten }\end{array}$ & $\begin{array}{l}\text { Förderung des } \\
\text { Tumorwachstums } \\
\text { aufgrund der } \\
\text { Wirkmechanismen } \\
\text { wahrscheinlich }\end{array}$ \\
\hline Nodularin & $\begin{array}{l}\text { Zyklische } \\
\text { Peptide mit } \\
\text { ADDA }\end{array}$ & $\begin{array}{l}\text { Ähnlich wie Micro- } \\
\text { cystin }\end{array}$ & Keine Daten & Keine Daten & $\begin{array}{l}\text { Blockiert Proteinphospha- } \\
\text { tasen } 1 \text { und } 2 A \text { insb. in der } \\
\text { Leber } \\
\rightarrow \text { Zerstörung des Leberge- } \\
\text { webes, inneres Verbluten }\end{array}$ & Karzinogen \\
\hline $\begin{array}{l}\text { Cylindro sper- } \\
\text { mopsin }\end{array}$ & Alkaloid & $\begin{array}{l}2100(24 h) \\
200(5-6 d)\end{array}$ & $4400-6900 ?$ & 30 & $\begin{array}{l}\text { Blockiert Protein-Synthese; } \\
\text { erhebliche kumulative } \\
\text { Toxizität } \\
\rightarrow \text { Leberversagen }\end{array}$ & $\begin{array}{l}\text { Hinweis auf Karzi- } \\
\text { nogenität }\end{array}$ \\
\hline Anatoxin-a & Alkaloid & 250 & $\begin{array}{l}\text { Ca. } 2400 \\
\text { (erste Hinweise) }\end{array}$ & $\begin{array}{l}\text { Ca. } 100-2400 \\
\text { (erste Hinweise) }\end{array}$ & $\begin{array}{l}\text { Blockiert post-synaptische } \\
\text { Depolarisation und somit } \\
\text { neuronale Signalüber- } \\
\text { tragung } \\
\rightarrow \text { Atmungslähmung }\end{array}$ & Keine Daten \\
\hline $\begin{array}{l}\text { Saxitoxine und } \\
\text { Strukturanaloge } \\
\text { (auch als „PSP-To- } \\
\text { xine, d. h. „Paralytic } \\
\text { Shellfish Poisons" } \\
\text { bekannt) }\end{array}$ & Alkaloid & $10-30$ & $128-420$ & Keine Daten & $\begin{array}{l}\text { Blockieren Natrium-Kanäle } \\
\text { und somit neuronale Sig- } \\
\text { nalübertragung } \\
\rightarrow \text { Atmungslähmung }\end{array}$ & Keine Daten \\
\hline Anatoxin-a (S) & $\begin{array}{l}\text { Organo- } \\
\text { phosphat }\end{array}$ & 20 & Keine Daten & Keine Daten & $\begin{array}{l}\text { Blockiert Acetylcholineste- } \\
\text { rase und somit neuronale } \\
\text { Signalübertragung } \\
\rightarrow \text { Atmungslähmung }\end{array}$ & Keine Daten \\
\hline
\end{tabular}

ADDA 2S,3S,8S,9S)-3-amino-9. methoxy-2,6,8-trimethyl-10-phenyldeca-4,6-dienoic acid ist eine Aminosäure, die spezifisch für Microcystine ist und bislang in keinem anderen Organismus gefunden wurde, NOAEL No observed advers effect level, LOAEL Lowest observed advers effect level, i.p. intra peritoneal.

wurde [7]. Einschränkend muss aber festgehalten werden, dass in diesen Oberflächenwässern gleichzeitig weitere lebertoxische Substanzen vorlagen und z. B. die endemische Rate an Hepatitis B in der Region hoch war. Die International Agency for Research on Cancer (IARC) hat Microcystin-LR in die Gruppe 2B („possibly carcinogenic to humans") eingestuft [7].

Bei einem Vorfall in Brasilien wurde mit Microcystinen und vermutlich auch mit weiteren Cyanotoxinen (insb. Cylindrospermopsin) kontaminiertes Wasser zur Hämodialyse eingesetzt, woraufhin die behandelte Patientengruppe typische akute Vergiftungserscheinungen zeigte, die bei über 70 Patienten zum Tod führten [8]. Eindeutig belegte Todesfälle durch Microcystin-Aufnahme beim Baden oder anderweitiger Freizeitnutzung von Gewässern mit „Blaualgenblüten“ sind bislang nicht bekannt; Microcystinkonzentrationen erreichen jedoch in ausgeprägten „Blüten“ Bereiche von mehr als 10 mg/L, die im Vergleich zu den Ergebnissen von Tierexperimenten mit oraler Dosierung das Risiko einer akuten Vergiftung nicht ausschließen lassen, insbesondere wenn von solchem Wasser mehr als 20-100 mL verschluckt werden ([9]; siehe unter Abschn. 1.4).

\subsection{Cyanobakterien und Cyanotoxine - Vorkommen und Exposition}

Trotz Verbesserungen gegenüber der Eutrophierungssituation vor 20 Jahren ist in vielen bundesdeutschen Badegewässern eine Sichttiefe von $1 \mathrm{~m}$ nach wie vor nicht ständig eingehalten. Häufig sind Massenentwicklungen von Cyanobakterien Hauptursache dieser Trübung. Ursache dieser Massenentwicklungen ist die Überdüngung der Gewässer (Stickstoff und Phosphor) aus Klärwerksabläufen, aus Abschwemmungen von landwirtschaftlich genutzten Flächen sowie in 


\begin{tabular}{|c|c|}
\hline Cyanobakterien & Toxine \\
\hline Anabaena (jetzt Dolichospermum) & $\begin{array}{l}\text { Microcystine, Anatoxine, Cylindrospermopsine, Sa- } \\
\text { xitoxine }\end{array}$ \\
\hline Aphanizomenon inkl. Cuspidothrix & $\begin{array}{l}\text { Anatoxine, Cylindrospermopsine, Saxitoxine, Micro- } \\
\text { cystine }^{\mathrm{a}}\end{array}$ \\
\hline Cylindrospermopsis raciborskii & Cylindrospermopsine, Saxitoxine \\
\hline Lyngbya & Saxitoxine \\
\hline Microcystis & Microcystine, Anatoxine ${ }^{a}$ \\
\hline Nodularia spumigena & Nodularine \\
\hline Nostoc & Microcystine, Nodularine ${ }^{a}$ \\
\hline Oscillatoria & Anatoxine, Saxitoxine \\
\hline Planktothrix (P. agardhii, P. rubescens) & Microcystine, Anatoxine ${ }^{a}$ \\
\hline Phormidium & Anatoxine, Microcystine \\
\hline Raphidiopsis & Cylindrospermopsine, Saxitoxine ${ }^{a}$ \\
\hline
\end{tabular}

Städten auch aus der Misch- und Trennkanalisation und von versiegelten Flächen nach Starkregenereignissen.

In erster Linie bestimmt die Konzentration an Pflanzennährstoffen (in der Regel des Gesamtphosphors, auch „Gesamt-P“ genannt) die maximal mögliche Zelldichte der Cyanobakterien und somit auch die Toxinkonzentration. Die Relation zwischen Cyanobakterienmenge und Gesamt-P ist zwar auch vom Gewässertyp abhängig, im Allgemeinen kann jedoch davon ausgegangen werden, dass unterhalb einer Gesamt-P-Konzentration von ca. 0,02 mg/L hohe Biomassen an Cyanobakterien unwahrscheinlich sind, da die Kapazität für die Bildung hoher Zelldichten zu gering ist. Dies lässt sich aus dem P-Bedarf der Biomasse ableiten und wurde durch statistische Auswertungen von Daten aus rund 200 bzw. 800 europäischen Gewässern bestätigt [10, 11]. Umgekehrt zeigen diese Publikationen, dass im Bereich von 0,02-0,1 mg/L Gesamt-P die Wahrscheinlichkeit größerer Cyanobakterien-Vorkommen zunimmt, insbesondere ab 0,05 mg/L Gesamt-P, wobei jedoch andere Faktoren wie die Wasseraufenthaltszeit, Schichtung, etc. beeinflussen, ob Cyanobakterien oder ggf. andere Algen zur Dominanz gelangen.

In schnell fließenden Gewässern (Wasseraufenthaltszeit $<3$ Tage) haben Cyanobakterien keine ausreichend stabilen Bedingungen für ihre Vermehrung bis hin zur Massenentwicklung. Anders verhält es sich in Flüssen mit längeren Ab- schnitten geringer Strömung (z. B. Flußseen mit einer Wasseraufenthaltszeit von 3-30 d): dort sind die Bedingungen ausreichend lange stabil, dass sich bei hohen Nährstoffgehalten manche Arten wie die potentiell toxische Planktothrix agardhii stark vermehren, zu Sichttiefen <0,5 Metern und einem Risiko der Toxin-Exposition führen.

$\mathrm{Zu}$ Massenentwicklungen neigen in stehenden, nährstoffreichen Gewässern vor allem die potentiell toxischen Cyanobakterien der Gattungen Microcystis, Planktothrix, Anabaena und Aphanizomenon (•Tab. 2), insbesondere während der Sommermonate. Beim Vorkommen von Microcystis und Planktothrix werden nahezu immer die hepatotoxischen Microcystine nachgewiesen, bei Aufrahmungen von Microcystis am Ufer oftmals in hohen Konzentrationen bis in den Bereich von mg/L [5, 12]. An der Ostseeküste kann bei massenhaftem Auftreten von Nodularia spumigena das in Struktur und Wirkung den Microcystinen sehr ähnliche Nodularin (siehe - Tab. 1) ebenfalls zu einem Expositionsrisiko bei Freizeitaktivitäten führen. Auch Cylindrospermopsin wurde in Deutschland häufig nachgewiesen, jedoch bislang nicht in gesundheitsrelevanten Konzentrationen [4]. Neurotoxine wurden bislang in Deutschland seltener und nur in geringen Konzentrationen gefunden. Jedoch können hohe Konzentrationen von Cylindrospermopsin und Neurotoxinen (Anatoxine, Saxitoxine) bei Massenentwicklungen potenziel- ler Produzenten der Gattungen Aphanizomenon und Anabaena nicht ausgeschlossen werden $[4,13]$. Da die akute Toxizität der meisten Neurotoxine stärker ausgeprägt ist als die der Microcystine (siehe Tab. 1) und auch tödliche Cyanotoxin-Vergiftungen von Vieh und Wildtieren häufiger auf Neurotoxine zurückgeführt wurden, ist das Risiko akuter Vergiftungen bei toxin-haltigen „Blüten“ dieser Arten ggf. höher.

Neben den oben beschriebenen planktisch (d. h. frei im Wasser) lebenden Cyanobakterien können auch einige der benthischen (d. h. festsitzenden) Cyanobakterien Toxine enthalten, vor allem aus den Gattungen Oscillatoria, Phormidium und Lyngbya (14, - Tab. 2). Sie wachsen als Matten in meist klaren (Fließ-)Gewässern auf dem Gewässergrund oder auf anderen Oberflächen. Festsitzende, untergetauchte Matten stellen vor allem eine Gefahr für trinkendes Vieh und für Haustiere dar. Lösen sie sich und treiben auf, so können sie auch eine Gefahr für Badende darstellen, wenngleich dies deutlich weniger wahrscheinlich ist als bei auftreibenden „Blüten“ planktischer Cyanobakterien. Da toxische benthische Cyanobakterien in Neuseeland häufig beobachtet werden, gehen diese dort auch in die Leitlinien zum Schutz vor toxischen Cyanobakterien ein [2]. Für Deutschland liegen bislang keine Untersuchungen zum Vorkommen von toxischen benthischen $\mathrm{Cy}$ anobakterien vor.

Zudem wurden auch bei anderen Cyanobakterien (z. B. Limnothrix, Anacystis) Hinweise auf Toxizität in Einzelfällen beobachtet, jedoch wurden die Toxine bislang nicht identifiziert. Andererseits wurden Microcystine in Freilandpopulationen mit Dominanz von z. B. Gloeotrichia, Woronchinia, Snowella nachgewiesen, für eine eindeutige Zuordnung der Toxinproduktion fehlen jedoch Nachweise in Kultur-Stämmen dieser Organismen.

Folgende Eigenschaften der Cyanobakterien stellen für die Überwachung des Cyanotoxin-Vorkommens besondere Herausforderungen dar:

- Die Toxingehalte in Cyanobakterien-Populationen können sehr unterschiedlich sein, da jede Population in unterschiedlichem Maß aus verschiedenen Genotypen zusammengesetzt 
ist, wobei manche Genotypen Toxine produzieren und andere nicht. Darüber hinaus können sich die toxinproduzierenden Genotypen sowohl hinsichtlich der synthetisierten Toxine (z. B. verschiedene Microcystinvarianten) als auch der Toxinmengen unterscheiden. Morphologisch (bei der mikroskopischen Untersuchung) sind diese Genotypen nicht zu unterscheiden. Anhand der Cyanobakterien-Konzentration (d. h. ihre Biomasse pro Liter) kann man daher das Expositionsrisiko nur als "worst case" Annahme abschätzen, indem man die für den schlimmsten Fall anhand von Literaturdaten maximal zu erwartenden Toxingehalte zugrunde legt. Dies ist im Sinne des vorsorgenden Gesundheitsschutzes, kann aber auch zu einer ggf. erheblichen Überschätzung des Risikos und folglich zu überzogenen Maßnahmen führen. Die Messung der tatsächlichen Microcystinkonzentration kann hier eine realistischere Bewertung ermöglichen.

- Das Cyanobakterien-Vorkommen kann im Gewässer räumlich und zeitlich aufgrund biotischer und abiotischer Einflüsse ein äußerst heterogenes Verteilungsmuster annehmen, insbesondere für diejenigen Arten und Gattungen, die aufgrund ihrer Gasvakuolen an der Wasseroberfläche zu so genannten „Wasserblüten " auftreiben oder „,aufrahmen“ (vor allem Microcystis, Anabaena, Nodularia). Diese „Blüten“ können windabhängig in bestimmten Gewässerbereichen akkumulieren, jedoch auch bei Wechsel der Windrichtung rasch wieder verdriftet werden. Somit beeinflussen auch physikalische Gewässereigenschaften wie die Morphologie und die Lage von Badebereichen das potenzielle Gesundheitsrisiko durch toxische Cyanobakterien. Da die „Blüten“ mit der Anreicherung ihrer Zellen um bis zu mehrere Größenordnungen zu sehr hohen Toxinkonzentrationen führen können, geht von ihnen das höchste Risiko aus. Andererseits ist dieses auch bereits mit bloßem $\mathrm{Au}$ ge zu erkennen und somit für informierte Badende vermeidbar.
- Auch in Gewässern mit geringer Gesamt-P-Konzentration $(<0,02 \mathrm{mg} / \mathrm{L})$ und somit geringer Zelldichte an Cyanobakterien können ebenso „Blüten“" oder „Schlieren“ an der Wasseroberfläche entstehen, wenn bei Dominanz von auftreibenden Arten diese aus einem sehr tiefen und großen Wasserkörper an der Wasseroberfläche akkumulieren oder durch Durchmischung an die Gewässeroberfläche gelangen wie z. B. Planktothrix rubescens in Voralpenseen. Jedoch sind diese Phänomene in der Regel räumlich und zeitlich stark begrenzt, und während der Sommermonate befindet sich Planktothrix rubescens in tiefen, den Badegästen nicht zugänglichen Wasserschichten.

- Die Cyanobakterien-Population in einem Gewässer variiert im jahreszeitlichen Muster in Abhängigkeit von abiotischen (Temperatur, Lichtverfügbarkeit) und biotischen (Konkurrenz durch andere Phytoplankton-Arten, Fraßdruck durch Zooplankton, Konkurrenz durch Wasserpflanzen) Faktoren. In manchen Gewässern (vorwiegend in größeren) wiederholen sich relativ ähnliche jahreszeitliche Muster von Jahr zu Jahr, sodass bei mehrjähriger Kenntnis des Gewässers recht gute Prognosen des Vorkommens möglich sind. In anderen (insb. kleinen Seen, Flußseen mit wechselnden Aufenthaltszeiten) sind die Muster des Cyanobakterien-Vorkommens stärker variabel.

Für die Bewertung des Expositionsrisikos sind aufgrund dieser Zusammenhänge Kenntnisse des Gewässers unabdingbar. Durch gute Kenntnisse des Gewässers und seines Einzugsgebietes kann eingeschätzt werden, ob das Auftreten hoher Cyanobakterien-Dichten aufgrund der chemisch-physikalischen Bedingungen überhaupt zu erwarten ist. Die Umsetzung der EG-Badegewässerrichtlinie liefert im Rahmen der Erstellung des Badegewässerprofils hierfür einen Ansatz, und die Wasserrahmenrichtlinie fordert für Seen $>50$ ha ebenfalls eine Beurteilung des Phytoplanktons. Für diese Gewässer können Gesundheitsämter daher in der Regel Daten von der zuständigen Umweltbehörde erbitten.

Nach Untersuchungen über einen Zeitraum von 2-3 Jahren mit 14-tägigem bis monatlichem Probenahmeraster kann gut beurteilt werden, ob Cyanobakterien zu erwarten sind. Ist dies der Fall, so ist die Kenntnis der vorherrschenden Gattungen zur Beurteilung der zu erwartenden Toxine sowie der Verteilung im Gewässer (z. B. aufrahmende Gattung oder nicht) für die Überwachung von großem Nutzen. Nach einem solchen intensiven Programm zur Bestandsaufnahme kann der Überwachungsaufwand häufig reduziert und gezielt auf die ggf. kritischen Zeiträume sowie Gewässer fokussiert werden. Die dafür notwendige Bewertung wird durch gewässerökologische Fachkompetenz wesentlich erleichtert. Insbesondere für die Phase der Planung von Untersuchungsprogrammen, der Etablierung der Methoden und der Bewertung der Ergebnisse empfiehlt sich die Kooperation der zuständigen Gesundheitsbehörde mit den regionalen Umweltbehörden und/oder mit anderen Institutionen, die das jeweilige Badegewässer unter ökologischen Gesichtspunkten untersuchen und bewerten.

\subsection{Gesundheitsgefährdung durch Cyanobakterien}

Nach direktem Kontakt mit Cyanobakterien wurde verschiedentlich über lokale Symptome wie Haut-/Schleimhautreizungen, Bindehautentzündungen und Ohrenschmerzen berichtet. Als Ursache hierfür werden weniger die o. g. Cyanotoxine, sondern vielmehr andere Zellbestandteile oder Bakterien in den Schleimhüllen von Cyanobakterien diskutiert. Allerdings gibt es für Cylindrospermopsin im Tierversuch Hinweise auf irritierende und sensibilisierende Eigenschaften bei Hautkontakt, wenn auch die eingesetzten Mengen weit über den in Gewässern üblicherweise auftretenden Mengen lagen [15]. Auch allergische Reaktionen auf Cyanobakterien werden eher auf andere Zellbestandteile oder Begleitbakterien zurückgeführt, da z. B. Microcystin-LR im Tierversuch nur ein sehr geringes allergenes Potential zeigte [16]. Insgesamt findet man in jüngeren, gezielten Studien eher wenige Hinweise auf eine weite Verbreitung der in frühe- 
ren Kasuistiken beschriebenen Haut- und Schleimhautreizungen sowie von allergischen Reaktionen, jedoch scheinen Menschen mit allergischer Disposition ein höheres Risiko zu haben [17].

Darüber hinaus wurden auch schwerer wiegende gesundheitliche Beeinträchtigungen wie Gastroenteritiden, Atemwegserkrankungen und Leberveränderungen dem Kontakt mit Cyanobakterien zugeschrieben [18]. Die Mehrzahl der letztgenannten Beobachtungen wurde im $\mathrm{Zu}$ sammenhang mit dem Genuss von kontaminiertem Trinkwasser dokumentiert. Bei diesem Expositionspfad werden aufgrund der größeren und geschlosseneren Kollektive Auffälligkeiten eher erkannt als bei Badenden.

Zur Exposition beim Baden liegen, neben einzelnen Kasuistiken, mehrere epidemiologische Untersuchungen vor [19-22]. Im Gesamtbild dieser Studien ergibt sich, dass durch die Exposition gegenüber Cyanobakterien im Rahmen der Freizeitnutzung von belasteten Gewässern gelegentlich leichte, vorrübergehende Krankheitserscheinungen gastrointestinaler, dermaler und respiratorischer Art auftreten können. Es bestand kein Zusammenhang zu den Konzentrationen an bekannten Cyanotoxinen (v. a. Microcystine), wohl aber zur Menge an Cyanobakterien im Gewässer. Die Schwellenwerte des Cyanobakterien-Biovolumens, bei denen die Symptome signifikant häufiger auftraten, lagen je nach Studie bei ca. 2 bis $11 \mathrm{~mm}^{3} / \mathrm{L}$ (Biovolumen für Microcystis, zur Vergleichbarkeit berechnet aus Angaben der Originalarbeiten). Bei diesen Betrachtungen ist jedoch zu berücksichtigen, dass sie systemische Wirkungen nach oraler Aufnahme nur bedingt erfassen können. Zum einen ist die Anzahl der Personen, die große Wassermengen aufnehmen, eher zu gering, um in einem größeren Personenkollektiv statistisch signifikant aufzufallen; zum anderen waren die Untersuchungszeiträume der epidemiologischen Studien zu kurz, um längerfristige Wirkungen zu erfassen.

Durch verbreitete Information, Aufklärung und Warnung mag die Freizeitnutzung von Gewässern bei deutlich sichtbaren „Algenblüten“ zurückgegangen sein; sie ist trotzdem weiterhin verbreitet, auch bei stark ausgeprägten Blüten, scheinbar ohne dass Auffälligkeiten bekannt würden. Jedoch hat in Finnland die zuständige Gesundheitsbehörde in Sommern mit erhöhtem Vorkommen von Cyanobakterien 3 bis 4-fach häufigere telefonische Berichte über gesundheitliche Beeinträchtigungen nach dem Baden entgegengenommen als in anderen Jahren [23].

Für den Gesundheitsschutz gilt es zu unterscheiden zwischen den leichten, vorübergehenden und (trotz statistischer Signifikanz) eher selten auftretenden Wirkungen einerseits und den systemischen Wirkungen der Cyanotoxine nach oraler Aufnahme andererseits. Letzeren kommt die größte Bedeutung zu. Dies zeigen zahlreiche Fallbeispiele verendeter Wildund Haustiere, die größere Mengen an Cyanobakterien aufgenommen haben und typische Symptome einer Microcystin- oder Neurotoxin-Intoxikation (v. a. Anatoxin-a) aufweisen [24-26]. Auch Berechnungen anhand der toxikologischen Ergebnisse von Tierversuchen mit Hilfe von Annahmen über die oral aufgenommene Wassermenge verdeutlichen dieses Risiko [27, 28]. Eine besondere Risikogruppe unter Badegästen sind hierfür im Uferbereich der Badegewässer spielende Kleinkinder im Krabbelalter aufgrund ihres Spielverhaltens und durch ihren häufigen Hand-Mund-Kontakt, bei dem sie unbeabsichtigt größere Mengen an mit $\mathrm{Cy}$ anobakterien belastetem Sand und Wasser aufnehmen können. Auch können ältere Kinder und Jugendliche beim Toben im Flachwasserbereich oder beim Schwimmen lernen größere Wassermengen aufnehmen, und auch bei Sportarten bei denen es zu einem intensiven Wasserkontakt kommen kann, wie Windsurfen, Wasserskifahren und ggf. auch beim Segeln, besteht ein erhöhtes Risiko für eine orale Aufnahme [29]. Die größten Wassermengen werden jedoch vermutlich bei Badeunfällen („Beinahe-Ertrinken“) aufgenommen. In der Konsequenz einer Gesamtbetrachtung dieser Erkenntnisse gilt es, vor oraler Aufnahme zu schützen ohne die Freizeitnutzung der Gewässer stärker einzuschränken, als aufgrund des Gesundheitsrisikos angemessen ist.

Für eine Betrachtung der Risiken und Beeinträchtigungen durch Cyanobakterien-Massenentwicklungen sind somit fol- gende Gesundheitsaspekte einzubeziehen:

- Systemische Wirkungen durch Cyanotoxine,

- Risiko durch die ausgeprägte Gewässertrübung bei hohem Cyanobakterien-Vorkommen bei Badeunfällen Ertrinkende nicht sehen zu können,

- Gelegentlich vorkommende, leichte, vorrübergehende gastrointestinalen, dermalen und respiratorischen Krankheitserscheinungen sowie Haut- und Schleimhautreizungen; ggf. auch allergische Reaktionen sensibler Personen.

\subsection{Risikoabschätzung und Leitwerte}

Eine Risikoabschätzung für Freizeitaktivitäten ist aufgrund der eingeschränkten toxikologischen und epidemiologischen Datenlage mit Unsicherheiten verbunden. Der o. g. derzeitige Kenntnisstand zeigt jedoch, dass Baden in eutrophen, stark mit toxischen Cyanobakterien belasteten Gewässern ein Gesundheitsrisiko darstellt, sofern es mit oraler Exposition verbunden ist, insbesondere wenn diese innerhalb weniger Tage bis Wochen mehrfach wiederholt auftritt. Dies gilt ebenso für andere Wassersportarten mit hohem Expositionsrisiko (z. B. Segeln, Tauchen, Windsurfen).

Heute verwenden die meisten Staaten als Grundlage für Leitwerte zum Schutz der Gesundheit vor Cyanotoxinen ein Maß der Cyanobakterienbiomasse - d. h. die Zelldichte, das Biovolumen (BV) oder die Konzentration des Pigments Chlorophyll-a (Chl.-a). Die Werte für Cyanobakterienbiomasse leiten sich dabei oft von den regional zu erwartenden Toxingehalten pro Biomasseeinheit ab. Die Regelungen vieler Staaten enthalten zudem einen Microcystin-Höchstwert als Orientierung oder Auslöser für die höchste Alarmstufe [2]. Meist orientieren sich die Regelungen an dem dreistufigen Überwachungskonzept der WHO, deren unterste Warnstufe jedoch eher die irritierenden oder allergischen Reaktionen durch Cyanobakterien heranzieht und aus epidemiologischen Daten Zellzahlen von 20.000 Zellen/ml (oder $10 \mu \mathrm{g} / \mathrm{L}$ Chl.-a oder Biovolumen von $\sim 2 \mathrm{~mm}^{3} / \mathrm{L}$ für Microcystis) ab- 


\begin{tabular}{|c|c|c|c|}
\hline & Zellzahl (Biovolumen) & $\begin{array}{l}\text { Chl.-a (bei Dominanz von } \\
\text { Cyanobakterien) }\end{array}$ & Microcystin konzentration \\
\hline $\begin{array}{l}\text { Geringe Wahrscheinlichkeit adver- } \\
\text { ser Wirkung auf die Gesundheit }\end{array}$ & $\begin{array}{l}20,000 / \mathrm{mL} \\
\left(\sim 2 \mathrm{~mm}^{3} / \mathrm{L}\right)\end{array}$ & $10 \mu \mathrm{g} / \mathrm{L}$ & $\begin{array}{l}2-4 \mu \mathrm{g} / \mathrm{L} \\
\text { (maximal } 10 \mu \mathrm{g} / \mathrm{L} \text { ) }\end{array}$ \\
\hline $\begin{array}{l}\text { Moderate Wahrscheinlichkeit ad- } \\
\text { verser Wirkung auf die Gesundheit }\end{array}$ & $\begin{array}{l}100,000 / \mathrm{mL} \\
\left(\sim 10 \mathrm{~mm}^{3} / \mathrm{L}\right)\end{array}$ & $50 \mu \mathrm{g} / \mathrm{L}$ & $\begin{array}{l}20 \mu \mathrm{g} / \mathrm{L} \\
\text { (maximal } 50-100 \mu \mathrm{g} / \mathrm{L})\end{array}$ \\
\hline $\begin{array}{l}\text { Hohe Wahrscheinlichkeit adverser } \\
\text { Wirkung auf die Gesundheit }\end{array}$ & $\begin{array}{l}\text { Aufrahmende Massenentwicklung } \\
(\text { („Blüte“) }\end{array}$ & & \\
\hline
\end{tabular}

leitet (• Tab. 3, [19, 27, 28]). Die bei diesen Biomassen an Cyanobakterien zu erwartenden Microcystinkonzentrationen von $<10 \mu \mathrm{g} / \mathrm{L}$ stellen bei den Expositionsszenarien durch Baden kein gesundheitliches Risiko dar. Die Bewertungsgrundlage für die mittlere Stufe sind sowohl die möglicherweise häufigeren unspezifischen Symptome als auch das Risiko durch erhöhte Microcystinkonzentrationen, und hier leiten sich die Zellzahlen oder die Konzentration an Chlorophyll-a von der maximal zu erwartenden Microcystinkonzentration von etwa $20 \mu \mathrm{g} / \mathrm{L}$ (im Extremfall bis zu 50 oder gar $100 \mu \mathrm{g} / \mathrm{L}$ ) ab. Die höchste Stufe beruht auf höheren Konzentrationen und insb. auf geschlossenen Cyanobakterien-Aufrahmungen an der Wasseroberfläche („Blüten“), da deren Microcystinkonzentrationen mehrere mg/L betragen können [12].

Auch die in der vorliegenden Empfehlung verwendete Ableitung von Leitwerten für die Cyanobakterien-Biomasse in Badegewässern orientiert sich sowohl an den beobachteten unspezifischen Symptomen wie Schleimhautreizungen durch Cyanobakterien als auch am Microcystin, denn unter den bisher bekannten Cyanotoxinen geht in Badegewässern in unseren Breiten von Microcystinen (bzw. an Küstengewässern von Nodularinen) das größte Risiko für die menschliche $\mathrm{Ge}$ sundheit aus. Dies insbesondere wegen ihres häufigen Vorkommens, ihrer ausgeprägten Toxizität und den ggf. sehr hohen Konzentrationen bei aufrahmenden Gattungen wie Microcystis. Dabei schlägt die Empfehlung für die CyanobakterienBiomasse zwei verschiedene Parameter zur Auswahl vor, entweder das Biovolumen oder die Konzentration an - vorwiegend Cyanobakterien zuzuordnendem - Chlorophyll-a. In beiden Fällen beru- hen die Biomasse-Leitwerte zur Einordnung des Risikos bzw. für das Ergreifen von Maßnahmen auf Erfahrungswerten für die in der Regel maximal im Gewässer zu erwartende Relation von Microcystinen zu Chlorophyll-a bzw. zu Biovolumen. Um eine schnelle Bewertung vor Ort zu ermöglichen, wurden für die jeweiligen Leitwerte näherungsweise entsprechende Sichttiefen abgeleitet [30].

Bisher ging die UBA-Empfehlung nach Anhörung der BWK [1] davon aus, dass bei Konzentrationen im Wasser von unter $100 \mu \mathrm{g}$ Microcystin/L ein ausreichender Sicherheitsabstand vor der Gefahr einer akuten Vergiftung mit diesen Cyanotoxinen durch Wasserschlucken beim Baden besteht und ein Schutz auch bei wiederholter saisonaler Exposition gewährleistet ist. Allerdings vernachlässigte diese Betrachtung das deutlich geringere Körpergewicht von Kleinkindern - für diese sollten rund $30 \mu \mathrm{g} / \mathrm{L}$ nicht überschritten werden, geht man von einer täglich wiederholten saisonale Exposition mit einer oralen Aufnahme von $100 \mathrm{ml}$ Wasser aus (• Tab. 4, [31]). Wie der internationale Vergleich verdeutlicht, verwenden andere Länder ebenfalls Leitwerte von 20$30 \mu \mathrm{g} / \mathrm{l}$ für Microcystine in Badegewässern [2], insb. auch um sicher zu stellen, dass Kleinkinder hinreichend wirksam geschützt sind.

Für die Neurotoxine besteht die gesundheitliche Besorgnis vorwiegend in ihrer z. T. ausgeprägten akuten oralen Toxizität (• Tab. 1), weniger im Hinblick auf chronische Wirkungen. Toxikologisch gesicherte Leitwerte liegen für diese Toxine zwar nicht vor; aufgrund der vorhandenen toxikologischen Daten kann jedoch davon ausgegangen werden, dass der für eine täglich wiederholte saisonale Exposition für Microcystin abgeleite- te Wert von $30 \mu \mathrm{g} / \mathrm{L}$ einen ausreichenden Schutz vor den eher akuten Wirkungen der Neurotoxine bietet. Für Cylindrospermopsin schlagen Humpage und Falconer [32] für Trinkwasser einen ähnlichen Leitwert vor wie für Microcystin; daher kann auch für dieses Toxin in für Freizeit genutzten Gewässern von einem ausreichenden Schutz ausgegangen werden, sofern $30 \mu \mathrm{g} / \mathrm{L}$ nicht überschritten sind. Auch sind die maximalen Relationen von Toxin zu Biovolumen oder Chlorophyll-a für diese Toxine ähnlich wie für Microcystin.

- Tab. 4 gibt in Ergänzung zu der saisonal täglich tolerierbaren Microcystin-Aufnahme ferner an, ab welcher Dosis und Konzentration das Risiko einer akuten Schädigung bestehen kann, insb. wenn bei Badeunfällen größere Wassermengen aufgenommen werden (exemplarisch wird hier mit 0,25 L gerechnet). Diese Angaben dienen nicht vorsorgenden sondern nachsorgenden Maßnahmen, insb. der Orientierung für die nähere Beobachtung von Personen bei Besorgnis nach dem Schlucken größerer Mengen an Cyanobakterien. Sie gelten allerdings nur für Microcystin - bei cyanobakteriellen Neurotoxinen ist die akut kritische Dosis möglicherweise geringer.

Es ist zu beachten, dass die vorliegende Empfehlung vorwiegend auf den Schutz vor den systemischen Wirkungen durch Cyanotoxine abzielt. Es muss davon ausgegangen werden, wie auch die vorgenannten epidemiologischen Daten zeigen, dass andere Wirkungen auf den Menschen (z. B. Haut- und Schleimhautreizungen) mit den in dieser Empfehlung aufgeführten Überwachungsmaßnahmen nicht in jedem Fall ausgeschlossen werden können. 


\begin{tabular}{|c|c|c|c|c|c|}
\hline $\begin{array}{l}\text { Zeitliches Muster der Exposition } \\
\text { und daraus folgende tolerierbare } \\
\text { Aufnahme }\end{array}$ & $\begin{array}{l}\text { Annahmen; } \\
\text { Quellen }\end{array}$ & $\begin{array}{l}\text { Tolerierbare Aufnah- } \\
\text { me pro kg Körperge- } \\
\text { wicht }\end{array}$ & $\begin{array}{l}\text { Tolerierbare Aufnah- } \\
\text { me für Kleinkinder } \\
\text { von } 10 \mathrm{~kg}\end{array}$ & $\begin{array}{l}\text { Tolerierbare Aufnah- } \\
\text { me für Jugendliche/ } \\
\text { Erwachsene von } 60 \mathrm{~kg}\end{array}$ & $\begin{array}{l}\text { Tolerierbare } \\
\text { Konzentration im } \\
\text { Badegewässer }\end{array}$ \\
\hline $\begin{array}{l}\text { Saisonal täglich tolerierbar } \\
\text { Exposition an mehreren Tagen pro } \\
\text { Woche für mehrere Wochen wäh- } \\
\text { rend einer Cyanobakterien-Massen- } \\
\text { entwicklung } \\
\text { AF =0,8; Wasseraufnahme } 0,1 \mathrm{~L} / \mathrm{d}\end{array}$ & $\begin{array}{l}\text { NOAEL von } \\
40 \mu g / k g \\
\text { und Tag [33] }\end{array}$ & $\begin{array}{l}0,4 \mu \mathrm{g} \text { pro kg Körper- } \\
\text { gewicht und Tag (ext- } \\
\text { rapoliert vom NOAEL } \\
\text { mit Faktor } 100 \text { ) }\end{array}$ & $4 \mu \mathrm{g}$ pro Tag & $24 \mu \mathrm{g}$ pro Tag & $\begin{array}{l}\text { Erwachsene: } 192 \\
\text { Kleinkinder: } 32 \mu \mathrm{g} \\
\text { pro L }\end{array}$ \\
\hline $\begin{array}{l}\text { Akut tolerierbar } \\
\text { Einmalige Exposition (d. h. einmal } \\
\text { pro Saison) } \\
\text { AF }=1 \text {; Wasseraufnahme } 0,25 \mathrm{~L}\end{array}$ & $\begin{array}{l}\text { NOAEL von } \\
250 \mu \mathrm{g} / \mathrm{kg} \\
\text { und Tag [34] }\end{array}$ & $\begin{array}{l}2,5 \mu \mathrm{g} \text { pro kg Körper- } \\
\text { gewicht und Expositi- } \\
\text { on (extrapoliert vom } \\
\text { NOAEL mit Faktor } 100 \text { ) }\end{array}$ & $\begin{array}{l}25 \mu \mathrm{g} \text { für eine einmali- } \\
\text { ge Exposition }\end{array}$ & $\begin{array}{l}150 \mu \mathrm{g} \text { für eine einmali- } \\
\text { ge Exposition }\end{array}$ & $\begin{array}{l}\text { Erwachsene: } 600 \\
\text { Kleinkinder: } 100 \mu \mathrm{g} \\
\text { pro L }\end{array}$ \\
\hline
\end{tabular}

\section{2 Überwachung}

\subsection{Grundlage der Überwachung - Einschätzung des Potentials für das Vorkommen von Cyanobakterien}

Grundlage der Überwachung ist die Einschätzung des Potenzials eines Gewässers für Cyanobakterien-Vorkommen bzw. Massenentwicklungen. Diese Einschätzung ist Teil der im Badegewässerprofil geforderten Bewertung. Verschiedene Ansätze zur gewässerökologischen Beurteilung sind in den letzten Jahrzehnten entwickelt und optimiert worden.

Der Trophie-Index nach LAWA [30] klassifiziert stehende Gewässer (natürliche Seen, Baggerseen, Talsperren und Speicherseen) anhand der Trophie-Parameter Chlorophyll-a, GesamtphosphorKonzentration und der Secchi-Sichttiefe. Dies ermöglicht eine Einordnung der Trophie eines stehenden Gewässers in acht Klassen von oligo- bis hypertroph. Das neu abgestimmte Verfahren ist mit dem WRRL-konformen PhytoSee-Verfahren (Biokomponente Phytoplankton) harmonisiert [35].

Für Seen $>50$ ha und für die Küstengewässer können auch Daten, die zur Umsetzung der Wasserrahmenrichtlinie (WRRL [36]) oder Oberflächenwasserverordnung (OGewV [37]) erhoben wurden, zur Einschätzung des Potenzials für Cyanobakterien-Vorkommen herangezogen werden. Hierbei kommen neben chemisch-physikalischen in unterschiedlichem Maße auch biologische Qualitäts- komponenten wie Phytoplankton, Makrozoobenthos und Makrophyten zum Einsatz, wobei auch hier Leitwerte für die unterschiedlichen Gewässertypen festgelegt wurden [38].

Fehlen solche Daten, z. B. für kleine Gewässer, ist ein einfacher Einstieg in die Beurteilung des Potenzials für Cyanobakterien-Massenentwicklungen die Messung der Gesamtphosphor-Konzentration (mindestens 4 Proben in einem Jahr, eine bei Vollzirkulation, 3 zwischen Mai und Sep.). Ab 0,025-0,05 mg/L Gesamt-P muss in stehenden und in sehr langsam fließenden Gewässern mit erhöhten Mengen an Cyanobakterien gerechnet werden [10,11]. Dies gilt auch für Flachseen und Flussseen im "guten ökologischen Zustand“, der auch erhöhte Mengen an Cyanobakterien in diesen natürlicherweise nährstoffreichen Gewässern berücksichtigt.

Auch Beobachtungen des Gewässers aus vergangenen Jahren, seien es Schlieren, Ergebnisse mikroskopischer Untersuchungen sowie Berichte über vergiftete Tiere, können nützliche Hinweise zur ersten Einschätzung eines Gewässers hinsichtlich des Vorkommens von (ggf. toxischen) Cyanobakterien geben.

Letztlich sind gute Kenntnisse des Gewässers und seines Einzugsgebietes sowie Verschmutzungsursachen, vor allem aber der Nährstoffkonzentrationen (Phosphor und evt. auch Stickstoff), wichtig für die Planung und Durchführung der in der EG-Badegewässerrichtlinie geforderten Bewirtschaftungsmaßnahmen.

\section{2 Überwachungsschema und Leitwerte}

Die Basis des hier vorgeschlagenen Vorgehens zur Überwachung bildet die Einschätzung des Potenzials eines Gewässers für Cyanobakterien-Vorkommen. Darauf baut das folgende 3-stufige Überwachungs- und Bewertungsschema auf, dessen wichtigste Mittel die visuelle Inspektion bei der Begehung vor Ort, die Sichttiefe sowie die qualitative Untersuchung auf Cyanobakterien sind (• Abb. 1). Sind quantitative Daten erwünscht, z. B. zur belastbareren Begründung von Nutzungseinschränkungen, kann die Bewertung ergänzend mit Analysen des Cyanobakterien-Chlorophyll-a oder-Biovolumen durchgeführt werden, für die im vorliegenden Überwachungsschema ebenfalls Konzentrationsbereiche für die drei Bewertungsstufen angegeben sind:

1. „Erhöhte Aufmerksamkeit“: Weist die Bewertung des Badegewässers im Rahmen des Badegewässerprofils auf das Potenzial für erhöhte Cyanobakterienbiomassen hin, so ist bereits ab Sichttiefen $<2 \mathrm{~m}$ und dem Vorkommen von Cyanobakterien ab 1-3 mm³/L bzw. 3-10 $\mu \mathrm{g} / \mathrm{L}$ Chl.-a eine Erhöhung der Überwachungsdichte während der Badesaison angezeigt (Stufe: „Erhöhte Aufmerksamkeit"). Fachlicher Hintergrund für diese Stufe ist das Potenzial für eine rasche Zunahme der Cyanobakterien in stark eutrophen Gewässern - d. h. innerhalb von 1-2 Wochen kann es zur Massenentwicklung kommen. 


\begin{tabular}{|c|c|c|c|}
\hline Stufe & $\begin{array}{l}\text { Einschätzung durch } \\
\text { visuelle Prüfung } \\
\text { (Vor-Ort und } \\
\text { Mikroskopie) }\end{array}$ & Maßnahmen & $\begin{array}{l}\text { Alternative und/oder } \\
\text { ergänzende } \\
\text { Einschätzung } \\
\text { durch Analysen }\end{array}$ \\
\hline 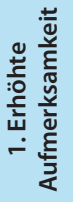 & $\begin{array}{l}\text { Trübung mit Sichttiefe } \\
<2 \mathrm{~m} \text { und } \\
\text { Vorkommen von } \\
\text { Cyanobakterien }\end{array}$ & $\begin{array}{l}\text { Überwachungshäufigkeit } \\
\text { während der Badesaison } \\
\text { ggf. verdichten; } \\
\text { Allgemeine Aufklärung } \\
\text { und Information über } \\
\text { dieses Badegewässer }\end{array}$ & $\begin{array}{l}>1 \mathrm{~mm}^{3} / \mathrm{L} \text { Cyanobakterien-BV } \\
\text { oder } \\
>5 \mu \mathrm{g} / \mathrm{L} \text { Cyanobakterien-Chl.a* }\end{array}$ \\
\hline 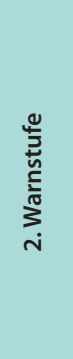 & $\begin{array}{l}\text { Schlieren durch } \\
\text { Cyanobakterien, jedoch } \\
\text { keine geschlossene } \\
\text { Schicht aufgerahmter } \\
\text { Cyanobakterien } \\
\text { oder } \\
\text { keine Schlieren, aber } \\
\text { grünliche Trübung } \\
\text { durch Cyanobakterien } \\
\text { mit Sichttiefe }<1 \text { m }\end{array}$ & $\begin{array}{l}\text { Warnhinweise (Wirkung } \\
\text { von Cyanobakterien, } \\
\text { Verhaltenshinweise) } \\
\text { für Badende (insb. Klein- } \\
\text { kinder) und wassersport- } \\
\text { treibende Personen } \\
\text { veröffentlichen }\end{array}$ & $\begin{array}{l}>3 \mathrm{~mm}^{3} / \mathrm{L} \text { Cyanobakterien-BV } \\
\text { oder } \\
>15 \mu \mathrm{g} / \mathrm{L} \text { Cyanobakterien-Chl.a* }\end{array}$ \\
\hline 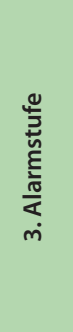 & $\begin{array}{l}\text { Große Bereiche mit } \\
\text { geschlossener Schicht } \\
\text { aufgerahmter Cyano- } \\
\text { bakterien ("Teppich") } \\
\text { oder } \\
\text { ausgeprägte grüne } \\
\text { Trübung durch } \\
\text { Cyanobakterien } \\
\text { mit Sichttiefe }<0,5 \mathrm{~m}\end{array}$ & $\begin{array}{l}\text { Warnhinweise (Wirkung } \\
\text { von Cyanobakterien, } \\
\text { Verhaltenshinweise) } \\
\text { für Badende (insb. Klein- } \\
\text { kinder) und wassersport- } \\
\text { treibende Personen } \\
\text { veröffentlichen; } \\
\text { Ggf. vorrübergehendes } \\
\text { Badeverbot bzw. Sperren** }\end{array}$ & $\begin{array}{l}>15 \mathrm{~mm}^{3} / \mathrm{L} \text { Cyanobakterien-BV } \\
\text { oder } \\
>75 \mu \mathrm{g} / \mathrm{L} \text { Cyanobakterien-Chl.a* }\end{array}$ \\
\hline
\end{tabular}

Abb. 1 Überwachungsschema für Bade- und Freizeitgewässer mit erhöhtem Potenzial für Cyanobakterien-Massenentwicklungen. Anmerkung: Eine Ergänzung der visuellen Prüfung durch Analysen erhöht die Belastbarkeit der Bewertung; Entscheidungen sind jedoch bereits auf der Grundlage der visuellen Prüfung möglich. *Dieser Wert gilt sowohl für direkt vor Ort bestimmte CyanobakterienChl. a Werte (mittels in situ Fluorometrie) als auch für nasschemische Bestimmungen bei ausgeprägter Cyanobakteriendominanz (die durch mikroskopische Untersuchung festgestellt wird). ${ }^{* *}$ Ausnahme: Wenn Microcystin bzw. Nodularin $<30 \mu \mathrm{g} / \mathrm{L}$ gelten hinsichtlich der Gefahren durch Cyanobakterien die Maßnahmen der Warnstufe; der Wert von $30 \mu \mathrm{g} / \mathrm{L}$ kann auch für die anderen Cyanobakterien-Toxine herangezogen werden
2. „Warnstufe“: Bei stärker ausgeprägter Trübung (Sichttiefe $<1 \mathrm{~m}$ ), lockeren Schlieren und Aufrahmungen durch Cyanobakterien oder Cyanobakterienbiovolumina ab 3-15 $\mathrm{mm}^{3} / \mathrm{L}$ bzw.

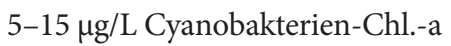
sollten Warnhinweise an die Bevölkerung gegeben werden. Für besonders empfindliche Personen ist das Auftreten von Haut- und Schleimhautreizungen in diesem Biomassebereich nicht auszuschließen, daher sollten diese entsprechend informiert sein. Auch können bereits Microcystinkonzentrationen im Bereich von $30 \mu \mathrm{g} / \mathrm{l}$ vorkommen.

3. „Alarmstufe“: Bei noch geringeren Sichttiefen $<0,5 \mathrm{~m}$ bzw. geschlossenen Teppichen an Cyanobakterien oder einem Cyanobakterienbiovolumen $>15 \mathrm{~mm}^{3} / \mathrm{L}$ bzw. $>75 \mu \mathrm{g} / \mathrm{L}$ Cyanobakterien-Chl.-a sollte ein vorübergehendes Badeverbot in Erwägung gezogen werden, $z$. B. wenn ein weite- res Anwachsen der Cyanobakterienbiomasse unmittelbar in den nächsten Tagen (z. B. aufgrund der Jahreszeit und/oder Wetterprognose) wahrscheinlich ist. Dies wird z. T. bereits aufgrund der Sicherheit (Sichtbarkeit Ertrinkender, die Rettung erst ermöglicht) praktiziert; das Risiko einer Exposition gegenüber hohen Microcystinkonzentrationen bei diesen Biomassen ist ein weiterer Grund für solche Maßnahmen.

Wenngleich die Leitwerte zur Abgrenzung dieser Stufen auf Erfahrungen zur Relation zwischen Cyanotoxin- und Biomassekonzentrationen basieren, sieht dieses dreistufige Schema zunächst keine Untersuchungen auf Cyanotoxingehalte vor. Diese kann jedoch als Erweiterung hinzugezogen werden: Bei geringen Sichttiefen und Vorkommen von Cyanobakterien, die eine Sperrung erfordern würden (Alarmstufe), kann durch den Nachweis von Microcystinkonzentratio- nen $<30 \mu \mathrm{g} / \mathrm{L}$ die Sperrung aufgehoben und zur Warnstufe zurückgekehrt werden. Falls Cyanobakterien-Arten massenhaft vorkommen, die vor allem Cylindrospermopsin sowie Neurotoxine produzieren (• Tab. 2), sollte als Entscheidungsgrundlage auch die Konzentration dieser Toxine bestimmt werden; dabei kann zur Ergebnisbewertung näherungsweise der Microcystin-Leitwert herangezogen werden. Da der Toxingehalt verschiedener Cyanobakterien-Populationen sehr unterschiedlich sein kann (siehe 1.2), können durch den Nachweis geringer Toxinkonzentrationen $(<30 \mu \mathrm{g} / \mathrm{L})$ die Badegewässer auch dann geöffnet bleiben, wenn Schlieren und Sichttiefen im Bereich von 0,5-1,0 $\mathrm{m}$ häufiger auftreten. Maßnahmen wie die Information der Badenden sollten dennoch getroffen werden und können auch die Sperrung des Badegewässers aus Sicherheitsgründen (Wasserrettung) beinhalten. 
Das vorgeschlagene Überwachungsschema, basierend auf der visuellen Inspektion sowie der Sichttiefe und einer qualitativen Untersuchung auf Cyanobakterien, setzt die Möglichkeit entweder einer Analyse am Mikroskop oder einer in situ Fluoreszenzmessung voraus. Es zeichnet sich durch eine günstige Relation von zeitlichem Aufwand gegenüber Aussagekraft der Ergebnisse aus.

Die visuelle Inspektion sowie die den Bewertungsstufen zugrundeliegenden Werte für Biovolumen bzw. Toxine können auch für die Überwachung von Badestränden der Küstengewässer angewendet werden. Die Überwachung der Küstengewässer auf das Vorkommen von Cyanobakterien-Massenentwicklungen erfordert jedoch ein weiträumigeres Vorgehen, um auch küstenferne Massenentwicklungen erkennen und rechtzeitig handeln $\mathrm{zu}$ können, falls entsprechende Wetterbedingungen diese an die Badestrände verdriften. Dies kann beispielsweise durch Fernerkundungen (u. a. mit FluoreszenzSensoren) im Rahmen von Überfliegungen erfolgen und im Rahmen des OstseeFährverkehrs wurden auch bereits Schiffe mit entsprechenden Sensoren ausgerüstet und die Daten kontinuierlich zur Auswertung übermittelt. Nicht zuletzt können Kontakte zu Nachbarländern frühzeitig Hinweise auf Cyanobakterien-Massenentwicklungen liefern.

\subsection{Methoden}

Im Folgenden werden ausschließlich Methoden für die Bewertung des aktuellen Cyanobakterien- sowie CyanotoxinVorkommens beschrieben. Auf Methoden zur gewässerökologischen Untersuchung sowie der Einschätzung des Potenzials für das Cyanobakterien-Vorkommen, wie beispielsweise die Bestimmung des Gesamtphosphorgehaltes [39], wird hier nicht näher eingegangen.

\subsubsection{Visuelle Inspektion}

Durch Ortsbegehungen können Cyanobakterien-Massenentwicklungen am unmittelbarsten erfasst und Maßnahmen umgehend eingeleitet werden. Bei Beobachtung von Aufrahmungen und Schlieren auf der Wasseroberfläche und/oder grünlicher Trübung des Wassers mit Sichttiefe unterhalb von $1 \mathrm{~m}$ ist jedoch zeitnah eine Wasserprobe mikroskopisch auf das Vorkommen von Cyanobakterien zu prüfen. Bestätigt sich der Verdacht auf Cyanobakterien als Ursache der Schlieren, so ist eine unverzügliche Information der Badenden (Warnhinweise in Medien und durch Informationstafeln vor Ort) angezeigt. Bei massivem Auftreten von „Wasserblüten“ sind Warnhinweise umgehend $\mathrm{zu}$ veröffentlichen und ggf. ein vorübergehendes Badeverbot zu erwägen. Bei Gewässern mit hohem Potenzial von Cyanobakterien-Vorkommen sollte bereits bei einer Sichttiefe von weniger als $2 \mathrm{~m} \mathrm{mi-}$ kroskopisch auf Cyanobakterien untersucht werden, um eine sich entwickelnde Population ggf. frühzeitig zu erfassen.

Bei einiger Erfahrung und Ortskenntnis können Probennehmer „Blüten“ auch ohne mikroskopische Untersuchung bereits mit bloßem Auge von anderen Anschwemmungen (z. B. von Wasserlinsen, Pollen) unterscheiden. Dann kann unter Umständen sofort gewarnt werden, z. B. durch Anbringen bereits mitgeführter Informationstafeln. Die mikroskopische Untersuchung auf Cyanobakterien sollte dennoch erfolgen.

Nicht alle Cyanobakterien-Arten treiben an der Wasseroberfläche auf. Insbesondere die für flache Gewässer charakteristische Planktothrix agardhii kann dennoch zu sehr hohen Zelldichten mit entsprechend stark ausgeprägter oliv-grünlicher oder blau-grünlicher Trübung anwachsen und zu hohen Toxinkonzentrationen führen.

Die Trübung wird als „Sichttiefe“ mit einer Secchi-Scheibe gemessen. Diese Scheibe ist von weißer Farbe (manche Modelle haben weiße und schwarze Segmente) und mit einigen Löchern versehen, um das Absenken im Wasser zu erleichtern. Ihr Durchmesser beträgt $20 \mathrm{~cm}$. An einer mit Dezimetereinteilung markierten Leine wird diese Scheibe im Gewässer hinabgelassen bis auf die Tiefe, in der ihr Umriss gerade nicht mehr sichtbar ist - die Sichttiefe. Vorzugsweise erfolgt diese Bestimmung vom Steg oder Boot aus, im Bootsschatten, um Störungen durch Spiegelungen zu vermeiden.
Zeitaufwand: Die visuellen Bewertung oder Inspektion erfolgt weitgehend vor Ort. Sowohl die Prüfung auf Schlieren oder Aufrahmungen an der Oberfläche als auch die Messung der Sichttiefe können innerhalb von wenigen Minuten vorgenommen werden. Die Untersuchung am Mikroskop, ob es sich bei den die Trübung oder Schlieren verursachenden Partikeln tatsächlich um Cyanobakterien handelt, erfolgt in der Regel erst im Labor und ist für eingearbeitete Mitarbeiter ebenfalls in wenigen Minuten durchführbar.

Erforderliche Ausrüstung: SecchiScheibe;

\subsubsection{Probenahme}

Die Beprobung von Gewässern für die Einschätzung des Potentials für Cyanobakterien-Vorkommen (WRRL, LAWA etc.) oder innerhalb umfassender Monitoringprogramme erfolgt in der Regel an der tiefsten Stelle eines Gewässers und besteht ggf. aus mehreren und/oder tiefenintegrierten Proben. Sie ist in Normen beschrieben (siehe auch [38]).

Für die Bewertung des Cyanobakterien-Vorkommens im Rahmen der Überwachung vor Ort werden Proben zur Bestimmung des Chlorophyll-a Gehaltes oder der Toxinkonzentration direkt im betroffenen Bereich des Badegewässers entnommen. Bei gleichmäßig im Wasser verteilten Cyanobakterien wird im Badebereich eine Schöpfprobe von der Wasseroberfläche entnommen. Bei Vorkommen von ausgeprägten Blüten mit aufrahmenden Schlieren oder teilweise oder ganz geschlossenen Teppichen sollte die Probe möglichst von der Stelle der größten Anhäufung von Cyanobakterien entnommen werden, um eine Exposition im Sinne eines „worst case “ zu erfassen. Auch hier erfolgt die Entnahme einer Schöpfprobe von der Wasseroberfläche. Ergänzend kann eine zweite Probe an einer Stelle mit gering ausgeprägter Aufrahmung entnommen werden, um die Spannbreite der Konzentrationen zu ermitteln.

Erforderliche Ausrüstung: Probenflaschen (für die Mikroskopie) für ein Volumen von 10-250 mL, ggf. Probenflaschen (1 L) für weitere Analysen (z. B. Chlorophyll-a, Toxine). 


\subsubsection{Qualitative Bestimmung von Cyanobakterien}

Bei Beobachtung von Aufrahmungen und Schlieren oder grünlicher Trübung während der Ortsbegehung klärt die mikroskopische Untersuchung der vor Ort entnommenen Proben, ob diese durch $\mathrm{Cy}$ anobakterien bedingt sind.

Mit dem Mikroskopieren vertraute Fachkräfte können Cyanobakterien nach kurzer Einarbeitungszeit rasch (d. h. in 5-10 min) erkennen und hinsichtlich ihrer Gattung taxonomisch einordnen. Häufig handelt es sich bei den „Wasserblüten" um die Gattungen Microcystis, Anabaena, Aphanizomenon flos-aquae, bei homogen verteilten Cyanobakterien um Planktothrix agardhii, Cuspidothrix (früher Aphanizomenon) issatschenkoi, Aphanizomenon sp. und bei rötlicher Färbung um Planktothrix rubescens.

\subsubsection{Quantitative Bestimmung von Cyanobakterien (Chlorophyll-a, Biovolumen)}

Die Menge an Cyanobakterien und Algen pro Liter Wasser kann anhand der Konzentration ihres wichtigsten Pigmentes, des Chlorophyll-a, näherungsweise abgeschätzt werden. Obwohl der Chlorophyll-a Gehalt in den Zellen und einzelnen Algenklassen etwas variiert, spiegelt er die Gesamtbiomasse von Algen und Cyanobakterien gut wider. Um ihn als Maß für die Cyanobakterien-Biomasse heranziehen zu können, muss allerdings durch die mikroskopische Analyse geklärt werden, ob Cyanobakterien überwiegen. Für eine gezieltere Bestimmung der Cyanobakterienmenge kann ihr spezifisches Pigment Phycocyanin hinzugezogen werden. Neben der nasschemischen Bestimmung im Labor stehen auch Sonden zur Verfügung, mit denen die Cyanobakterienbiomasse anhand der In-vivo Fluoreszenz dieser Pigmente direkt im Gewässer geschätzt wird.

Nicht zuletzt kann die Cyanobakterienbiomasse am Mikroskop anhand der Zelldichte bestimmt werden, aus der mithilfe des mittleren Zellvolumens das Biovolumen berechnet wird. Die Auswahl zwischen beiden Verfahren wird vom $\mathrm{Zu}$ griff auf die jeweilige Methode abhängig sein.
Nasschemische Bestimmung von Chlorophyll-a. 1 Liter Wasser wird in eine Flasche aus Glas oder Polyethylen abgefüllt. Diese wird dunkel gehalten und in einer Kühlbox gelagert.

Analyse: Die Probe sollte schnellstmöglich im Labor über Glasfaserfilter filtriert werden. Die Bestimmung der Chlorophyll-a-Konzentration erfolgt nach ISO 10260 [40], indem der Chlorophyll-a Gehalt der auf dem Filter zurückgehaltenen Algen- und Cyanobakterienzellen mit siedendem Ethanol extrahiert und seine Extinktion am Photometer bei $665 \mathrm{~nm}$ gemessen wird.

Zeitaufwand: Ein eingearbeitetes Labor kann ca. 25 Proben pro Tag analysieren. Die Extraktion muss mindestens über $6 \mathrm{~h}$ erfolgen.

Erforderliche Ausstattung: Filtrationsanlage; Abzug, Heizpilz und Kolben für die Extraktion; Zentrifuge oder Glasfaserfiltration zur Klärung des Extraktes; Photometer mit Wellenlängen von 665 und $700 \mathrm{~nm}$, Glasfaserfilter.

\section{Bestimmung von Chlorophyll-a durch In-vivo Fluoreszenz}

Für eine schnelle Bestimmung des Chlorophyll-a Gehaltes eignet sich die fluorometrische Chlorophyll-Analyse (In-vivo Fluoreszenz), bei der spezifisch die Konzentration des Cyanobakterien-Chlorophyll a sowie - je nach Ausstattung - auch verschiedener Algengruppen erfasst werden kann. Dadurch kann ggf. die Dominanz von Cyanobakterien bestätigt und als Cyanobakterien-Chlorophyll a bestimmt werden. Die Bestimmung kann im Labor erfolgen, portable Geräte erlauben auch eine Messung direkt im Gewässer. Diese Methode ist nicht genormt. Vergleichsuntersuchungen eines portablen Fluorometers (0-200 $\mu \mathrm{g}$ Chl.-a/L) mit mikroskopisch bestimmtem Biovolumen zeigten eine relativ gute Abschätzung der Cyanobakterienbiomasse. Jedoch scheint gerade für potentiell toxische Cyanobakterien die Übereinstimmung schlechter, insb. bei roter Pigmentierung (P. rubescens) und Koloniebildung (z. B. Microcystis, Aphanizomenon) [41]. Eine gelegentliche Überprüfung der Ergebnisse der In-vivo Fluoreszenz mit denen der Biovolumen-Bestimmung am Mikroskop (s. u.), um diese Signale für das Gewässer und die Situation typischen Cyanobakterienkonsortien zu „kalibieren“, kann die Quantifizierung erheblich verbessern. Diese Methode ist für eine schnelle Einschätzung der Situation vor Ort geeignet, insbesondere für die Erfassung der horizontalen und vertikalen Variabilität von Cyanobakterienblüten in einem Gewässer.

\section{Bestimmung des Biovolumens}

Die Bestimmung des Biovolumens erfolgt durch Auszählen einer mit Lugol'scher Lösung fixierten Probe (Probenvolumen: wenige $\mathrm{mL}$ ), in der Regel am Umkehrmikroskop bei 200- bis 400facher Vergrößerung [42].

Probenahme: $100 \mathrm{~mL}$ Wasser werden in eine Braunglasflasche abgefüllt, in der als Fixierungsmittel einige Tropfen konzentrierte Lugol'sche Lösung vorgelegt sind.

Zeitaufwand: Erfahrene Fachkräfte benötigen ca. $2 \mathrm{~h}$, bei artenreichen Proben ggf. bis zu $4 \mathrm{~h}$ pro Probe für das Biovolumen von Cyanobakterien.

Erforderliche Ausstattung: Mikroskop mit mindestens 200facher, besser mit 400facher Vergrößerung, vorzugsweise Umkehrmikroskop; Zählkammern.

\subsubsection{Quantifizierung der Micro- cystinkonzentration (und anderer (yanotoxine)}

Zur Bestimmung der Microcystinkonzentration haben sich 2 Verfahren bewährt, der Immunoassay (ELISA - Enzyme Linked Immuno Sorbent Assay) und die chemische Analyse mittels chromatographischer Trennung (HPLC - High Performance Liquid Chromatography) und Detektion der Microcystine anhand ihrer charakteristischen UV-Absorptionsspektren mittels Photodioden-Array-Detektor (PDA) oder Massenspektrometer. Für die Badegewässerüberwachung eignet sich der ELISA insbesondere für Behörden ohne einfachen Zugriff auf HPLC-Analytik, da er einfach zu handhaben, rasch durchzuführen ist und insb. bei Konzentrationen über $1 \mu \mathrm{g} / \mathrm{L}$ Ergebnisse liefert, die i. d. R. gut mit denen der HPLC-Analytik übereinstimmen [43]. Für einen schnellen und einfachen, jedoch nur halb-quantitativen Nachweis von Microcystinen sind seit kurzem Teststreifen auf dem Markt, 
die ebenfalls eine ausreichende Orientierung ermöglichen [44].

\section{ELISA für die Microcystinbestim-}

mung. ELISA-Kits für Microcystine werden kommerziell vertrieben. Vorteil des ELISAs ist seine rasche und recht einfache Durchführbarkeit anhand einer Schöpfprobe. Hierbei sollte darauf geachtet werden, dass es sich um einen sog. ADDA-ELISA handelt, da dieser die meisten der verschiedenen Microcystin-Strukturvarianten ausreichend gut erfasst. Grundsätzlich ist der ELISA mit einem relativ geringen apparativen Aufwand und geringen Kosten pro Probe (bei Probenserien) verbunden. Die Nachweisgrenze liegt bei $0,1-0,5 \mu \mathrm{g} / \mathrm{l}$.

Probenahme: Es genügen wenige $\mathrm{mL}$ einer Schöpfprobe, die dunkel und kühl $\mathrm{zu}$ lagern ist und wenige Stunden nach der Probenahme einzufrieren ist. In der Regel wird mit dem ELISA die Gesamtmicrocystinkonzentration bestimmt, die Bestimmung von gelöstem Microcystin erfordert eine Filtration der Probe vor Einfrieren (Glasfaser- oder Membranfilter).

Analyse: Durch 2 fach wiederholtes Auftauen und wieder Einfrieren werden möglichst alle Zellen aufgeschlossen und das vorwiegend intrazellulär vorliegende Microcystin freigesetzt. Die Durchführung der Analyse erfolgt nach den Angaben des Herstellers.

Zeitaufwand: Mit ELISA vertraute Fachkräfte benötigen 1,5-2 h für die Durchführung des ELISAs (ohne Probenvorbereitung wie Einfrieren/Auftauen).

Erforderliche Ausstattung: ELISA-Reader; ELISA-Kits, Pipetten.

Die instrumentelle Analytik von Microcystinen. Die instrumentelle Analytik von Microcystinen ist durch den Mangel an kommerziell erhältlichen Referenzsubstanzen erschwert. Es gibt zwar mittlerweile verschiedene Microcystine als Reinsubstanzen, jedoch sind die wenigsten davon zertifizierte Referenzstandards und zudem kommen diese Microcystine vor allem bei den Gattungen Microcystis und Anabaena vor. Charakteristische Microcystine der Gattung Planktothrix sind nur vereinzelt erhältlich.

Die Analytik von Microcystinen mittels HPLC-PDA ist in ISO 20179 be- schrieben [45]. Diese gilt nur für die Bestimmung der Strukturvarianten MC-RR, -YR und -LR, die vor allem bei Microcystis und Anabaena vorkommen, nicht jedoch bei Planktothrix agardhii bzw. rubescens. Allerdings besteht mit HPLC-PDA der Vorteil, Microcystine anhand ihres charakteristischen UV-Spektrums als solche identifizieren zu können und insbesondere die Möglichkeit der Quantifizierung auch von Strukturvarianten, für die keine Referenzsubstanzen auf dem Markt zur Verfügung stehen, da die bislang bekannten Absorptionskoeffizienten von verschiedenen Microcystinen sehr ähnlich sind [5]. Zur Quantifizierung kann ein Microcystin-LR-Standard verwendet und die Summe aller Strukturvarianten als Microcystin-LR-Konzentrationsäquivalente angegeben werden. Dies liefert eine Maximalabschätzung des Risikos, da Microcystin-LR als toxischste Strukturvariante zählt.

Im Gegensatz dazu können mittels Massenspektrometrie zwar die einzelnen Strukturvarianten identifiziert werden $[46,47]$, jedoch nur diejenigen Microcystine quantifiziert werden, von denen Referenzsubstanzen zur Verfügung stehen. Da dies jedoch nur für einen Teil der am häufigsten vorkommenden Microcystine der Fall ist (s. o.), führt diese Methode ggf. zu einer Unterschätzung der tatsächlichen Microcystinkonzentration.

Sowohl mit HPLC-PDA als auch mit LC-MS können Microcystinkonzentrationen von $1 \mu \mathrm{g} / \mathrm{L}$ sicher nachgewiesen werden, die Nachweisgrenzen hängen jedoch vom filtriertem Wasservolumen ab und können dadurch und bei LC-MS deutlich darunter liegen.

Die Nachteile der instrumentellen Analytik liegen im höheren Aufwand hinsichtlich apparativer Ausstattung und Erfahrung des Laborpersonals.

Probenahme: In der Regel liegt über $90 \%$ des Microcystins zellgebunden vor und wird nur bei Zelllyse freigesetzt, wobei es hier in der Regel rasch verdünnt und in der Regel innerhalb weniger Tage abgebaut wird. Für die Bestimmung des intrazellulären Microcystins wird $1 \mathrm{~L}$ Schöpfprobe entnommen, dunkel gelagert und sobald wie möglich über Glasfaseroder Membranfilter filtriert, bis sich ein deutlicher Belag auf dem Filter zeigt. Die
Filter werden tiefgefroren gelagert; über wenige Tage ist auch eine Lagerung hinreichend getrockneter Filter (Trocknung mindestens $24 \mathrm{~h}$ bei $40^{\circ} \mathrm{C}$ ) bei Raumtemperatur möglich, sofern die Filter wirksam vor Luftfeuchtigkeit geschützt werden. Für die Bestimmung von extrazellulärem Microcystin mittels HPLC-PDA sollte das Filtrat über Festphasenextraktion (SPE) aufkonzentriert werden, bei der Bestimmung mit LC-MS/MS kann das Filtrat meist auch ohne vorherige Aufkonzentrierung direkt analysiert werden.

Analyse: Die auf dem Filter zurückgehaltenen Zellen werden durch Einfrieren und Auftauen im feuchten Zustand aufgeschlossen und mehrfach mit 75\%igem Methanol extrahiert [5]. Nach Klärung der Extrakte durch Zentrifugation kann sich eine Festphasenextraktion anschließen oder unmittelbar die Analyse mittels HPLC-PDA oder LC-MS erfolgen. Bei der massenspektrometrischen Detektion hat sich die Analyse im multiple reaction monitoring (MRM) Modus bewährt [46, 47].

Erforderliche Ausstattung: Filtrationsanlage; Evaporator oder Speedvac zur Einengung der Extrakte; ggf. Anlage für Festphasenenxtraktion (SPE); HPLC-Anlage mit Pumpen zur Einstellung von Gradienten sowie PDA-Detektor/Massenspektrometer; ggf. Zentrifuge; Microcystin-LR.

Zeitaufwand: Ist abhängig von Erfahrung, Ausstattung, Art der Probenvorbereitung und Analytik, beträgt jedoch bei Standardverfahren mindestens 2 Tage.

Analytik anderer Cyanotoxine. Neben Microcystinen gibt es auch für Cylindrospermopsin und Saxitoxin kommerzielle ELISAs. Für Anatoxin-a ist ein Receptor-Binding Assay auf dem Markt, dessen Durchführung jedoch ein gut ausgestattetes Labor mit geschultem Personal voraussetzt.

Daneben können auch diese Toxine mittels HPLC-PDA, HPLC-FLD sowie LC-MS/MS identifiziert und quantifiziert werden. Aufgrund der eindeutigeren Identifizierung und Quantifizierung ist für Cylindrospermopsin und Anatoxin-a die massenspektrometrische Analyse zu bevorzugen [47, 48]. Die Nachweisgrenzen liegen je nach angereichertem Wasservolumen bzw. Zellen sowie Empfindlichkeit des Gerätes unter $1 \mu \mathrm{g} / \mathrm{L}$. Da 
Saxitoxine oft im Rahmen der Lebensmittelüberwachung von Muscheln bestimmt werden [49], empfiehlt es sich im Verdachtsfall die zuständige Lebensmittelbehörde vor Ort zu kontaktieren.

\subsection{Bewertung der Ergebnisse und Maßnahmen}

Im Vordergrund der Sofortmaßnahmen steht die Information der Öffentlichkeit durch Warnhinweise an den Badegewässern, im Internet sowie in den örtlichen Medien. Da Warnungen auf größere Akzeptanz stoßen, wenn sie auf einer regelmäßigen Information der Bürger über die Badegewässerqualität aufbauen können, empfiehlt sich eine regelmäßige Veröffentlichung der Messergebnisse nach der EG-Badegewässerrichtlinie unter Einbeziehung des Vorkommens von Cyanobakterien-Massenentwicklungen. Sofern eine starke Trübung durch hohe Zelldichten planktischer Algen verursacht ist, kann auch darüber informiert und im Hinblick auf Cyanobakterien Entwarnung gegeben werden.

Bei erhöhter Cyanobakterien-Dichte empfiehlt sich eine Intensivierung der Überwachung, da Cyanobakterien innerhalb kurzer Zeit (durch Aufrahmung innerhalb von Stunden, durch hohen Nährstoffgehalt innerhalb von Tagen) massive „Blüten“ bilden können. Bei starken Anschwemmungen auftreibender Cyanobakterien an Badebereichen und geringen Sichttiefen $(<50 \mathrm{~cm})$ ist ein vorübergehendes Badeverbot (bis zum Abklingen dieses Vorkommens) empfohlen (ggf. erfolgt dieses ohnehin aufgrund der hohen Trübung im Hinblick auf die Sicherheit der visuellen Überwachung der Badegewässer auf Badeunfälle).

Von Cyanotoxinen sind Badende deutlich geringer betroffen, wenn sie Bereiche besonders intensiver Cyanobakterien-Ansammlungen vermeiden. Häufig sammeln sich Zellen im Uferbereich an, sodass durch Schwimmen vom Steg oder Boot aus die Exposition gegenüber dem unmittelbaren Uferbereich erheblich reduziert werden kann. Aufgetriebene Cyanobakterien können jedoch ebenso „Teppiche" bilden, die weite Teile der Gewässeroberfläche bedecken, sodass auch Windsurfer und Segler bei häufigerem Kentern stark exponiert werden.
Wegen der ausgeprägten räumlichen und zeitlichen Variabilität des Cyanobakterien-Vorkommens ist eine besondere Flexibilität in ihrer Überwachung erforderlich. In stärkerem Maße als bei den für den Badenden „unsichtbaren“ Bakterien und Viren kann durch Information und Aufklärung die Eigenbeobachtung und der verantwortliche Umgang mit Cyanobakterien geschult werden.

Bei einer Sichttiefe von stets mindestens einem Meter in Gewässern erreicht die Cyanobakterien-Dichte selten ein gesundheitsgefährdendes Niveau, und bei Sichttiefen von $2 \mathrm{~m}$ sind Aggregationen von Cyanobakterien in gesundheitsgefährdender Dichte unwahrscheinlich. Ausnahmen können bei bestimmten Windverhältnissen in großen Gewässern auch bei Sichttiefen $>2$ m auftreten, in mesotrophen Gewässern sind solche Erscheinungen meist kurzzeitig und führen somit nicht zu einer langfristigen Exposition der häufig Badenden. Gesamtphosphor-Konzentrationen von 0,025$0,05 \mathrm{mg} / \mathrm{L} \mathrm{P}$ senken je nach Gewässertyp und Einzugsgebiet die Wahrscheinlichkeit des Auftretens von Cyanobakterien $[10,11,50]$.

Neben der von der EG-Badegewässerrichtlinie geforderten Risikoabschätzung durch Cyanobakterien-Massenentwicklung müssen auch geeignete Bewirtschaftungsmaßnahmen zu deren Vermeidung durchgeführt werden. Je nach Gegebenheiten vor Ort kann dies z. B. durch Sanierung der Abwassereinleitungen, angemessene Regenwasserbewirtschaftung von versiegelten Flächen, durch Änderung landwirtschaftlicher Praktiken und anderer Gewässerschutzmaßnahmen erreicht werden [50].

\section{Literatur}

1. Umweltbundesamt (2003) Empfehlung zum Schutz von Badenden vor Cyanobakterien-Toxinen. Bundesgesundheitsbl 46:530-538

2. Chorus I (2012) Current approaches to cyanotoxin risk assessment, risk management and regulation in different countries. Umweltbundesamt, Dessau

3. Badewasserrichtlinie (EG-BadewRL) Richtlinie 2006/7/EG des Europäischen Parlaments und des Rates vom 15 Februar 2006 über die Qualität von Badegewässern und deren Bewirtschaftung und zur Aufhebung der Richtlinie 76/160EWG
4. NOSTOTOX (2010) Abschlussbericht. http://www. kompetenz-wasser.de/index.php?id=382\&type $=0$ \&jumpurl=fileadmin\%2Fuser_upload\%2Fpdf\%2Fforschung\%2FNOSTOTOX\%2FNostotox_final_web.pdf.ZZugegriffen: 06. Mai 2015

5. Chorus I, Bartram J (1999) Toxic cyanobacteria in water: a guide to public health significance, monitoring and management. Für WHO durch E \& FN Spon/Chapman \& Hall, London. http://www.who. int/water_sanitation_health/resources/toxicyanbact/en/. Zugegriffen: 06. Mai 2015

6. Funari E, Testai E (2008) Human health risk assessment related to cyanotoxins exposure. Crit Rev Toxicol 38(2):97-125

7. IARC (2010) IARC monographs on the evaluation of carcinogenic risks to humans. Volume 94 . Ingested nitrate and nitrite, and cyanobacterial peptide toxins. World Health Organization (WHO) International Agency for Research on Cancer (IARC), Lyon

8. Jochimsen EM, Carmichael WW, An JS et al (1998) Liver failure and death following exposure to $\mathrm{mi}-$ crocystin toxins at a dialysis center in Brazil. N Eng Jed 338:873-878

9. Ibelings BW, Chorus I (2007) Accumulation of cyanobacterial toxins in freshwater "seafood" and its consequences for public health: a review. Environ Pollut 150(1):177-192

10. Chorus I, Niesel V (2011) Phytoplankton species response to oligotrophication. In: Chorus I, Schauser I (Hrsg) Oligotrophication of lake tegel and schlachtensee, Berlin. Analysis of system components, causalities and response thresholds compared to responses of other waterbodies. Umweltbundesamt, Dessau, S 22-58

11. Carvalho L, McDonald C, de Hoyos C et al (2013) Sustaining recreational quality of European lakes: minimizing the health risks from algal blooms through phosphorus control. J Appl Ecol 50:315323

12. Fastner J, Neumann U, Wirsing B, Weckesser J, Wiedner C, Nixdorf B, Chorus I (1999) Microcystins (hepatotoxic heptapeptides) in German fresh waters. Environ Toxicol 14:13-22

13. Bumke-Vogt C, Mailahn W, Chorus I (1999) Anatoxin-a and neurotoxic cyanobacteria in German lakes and reservoirs. Environ Toxicol 14:117-126

14. Catherine $Q$, Susanna W, Isidora ES, Mark H, Aurélie V, Jean-François H (2013) A review of current knowledge on toxic benthic freshwater cyanobacteria - ecology, toxin production and risk management. Water Res 47(15):5464-5479

15. Stewart I, Seawright AA, Schluter PJ, Shaw GR (2006) Primary irritant and delayed-contact hypersensitivity reactions to the freshwater cyanobacterium Cylindrospermopsis raciborskii and its associated toxin cylindrospermopsin. BMC Dermatol 6:5

16. Török A, Pálovics Á, Bánki M (2001) Allergenic (sensitization, skin and eye irritation) effects of freshwater cyanobacteria-Experimental evidence. Environ Toxicol 16:512-516

17. Stewart I, Robertson IM, Webb PM, Schluter PJ, Shaw GR (2006) Cutaneous hypersensitivity reactions to freshwater cyanobacteria - human volunteer studies. BMC Dermatol 6:6

18. Kuiper-Goodman T, Falconer I, Fitzgerald J (1999) Human health aspects. In: Chorus I, Bartram J (Hrsg) Toxic cyanobacteria in water: a guide to public health significance, monitoring and management. Chapman \& Hall, London, S 125-160 
19. Pilotto LS, Douglas RM, Burch MD, Cameron S, Beers M, Rouch GR et al (1997) Health effects of exposure to cyanobacteria (blue-green algae) due to recreational water-related activities. Aust N Z J Public Health 21:562-566

20. Stewart I, Webb PM, Schluter PJ, Fleming LE, Burns JW Jr, Gantar M, Backer LC, Shaw GR (2006) Epidemiology of recreational exposure to freshwater cyanobacteria - an international prospective cohort study. BMC Public Health 6:93

21. Backer LC, McNeel SV, Barber T et al (2010) Recreational exposure to microcystins during algal blooms in two California lakes. Toxicon 55(5):909921

22. Lévesque B, Gervais M-C, Chevalier P et al (2014) Prospective study of acute health effects in relation to exposure to cyanobacteria. Sci Tot Env 466467:397-403

23. Rapala J, Kilponen J, Järvinen M, Lahti K (2012) Finland: guidellines for monitoringo f cyanobacteria and their toxins. In: Chorus I (Hrsg) Current approaches to Cyanotoxin risk assessment, risk management and regulations in different countries. Umweltbundesamt, Dessau, S 54-62

24. Gugger $M$, Lenoir $S$, Berger $C$, Ledreux $A$, Druart J-C, Humbert J-F, Guette C, Bernard C (2005) First report in a river in France of the benthic cyanobacterium Phormidium favosum producing anatoxin-a associated with dog neurotoxicosis. Toxicon 45(7):919-928

25. Handeland K, Østensvik Ø (2010) Microcystin poisoning in roe deer (Capreolus capreolus). Toxicon 56(6):1076-1078

26. Backer LC, Landsberg JH, Miller M, Keel K, Taylor TK (2013) Canine cyanotoxin poisonings in the United States (1920s-2012): review of suspected and confirmed cases from three data sources. Toxins 5(9):1597-1628

27. Ian Falconer I, Bartram J, Chorus I, Kuiper- Goodman T, Utkilen H, Burch M, Codd GA (1999) Safe levels and safe practices. In: Chorus I, Bartram $J$ (Hrsg) Toxic cyanobacteria in water: a guide to their public health consequences, monitoring and management. Chapman \& Hall, London, S $161 \mathrm{ff}$

28. WHO (2003) Algae and cyanobacteria in fresh water. In: Guidelines for safe recreational water environments. Vol. 1: coastal and fresh waters. World Health Organization, Geneva

29. Giannuzzi L, Sedan D, Echenique R, Andrinolo D (2011) An acute case of intoxication with cyanobacteria and cyanotoxins in recreational water in Salto Grande Dam, Argentina. Marine Drugs 9(11):2164-2175

30. LAWA (2015) Trophieklassifikation von Seen; Richtlinie zur Ermittlung des Trophie-Index nach LAWA für natürliche Seen, Baggerseen, Talsperren und Speicherseen. Kulturbuch-Verlag, Berlin (im Druck)

31. Roller M (2000) Risikoabschätzung für die Exposition gegenüber Blaualgen (Cyanobakterien) und ihren Toxinen in Badegewässern. Gutachten erstellt für das Niedersächsische Ministerium für Frauen, Arbeit und Soziales, Hannover

32. Humpage AR, Falconer IR (2003) Oral toxicity of the cyanobacterial toxin cylindrospermopsin in male swiss albino mice: determination of no observed adverse effect level for deriving a drinking water guideline value. Environ Toxicol 18:94-103

33. WHO (1998) Guidelines for drinking water quality. 2 nd edition. Addendum to Vol 2 Health Criteria and other supporting information. WHO, Genf
34. Fromme H, Köhler A, Krause R, Führling D (2000) Occurence of cyanobacterial toxins - microcystins and anatoxin-a - in Berlin water bodies with implications to human health and regulations. Environ Toxicol 15:120-130

35. Mischke U, Riedmüller U, Hoehn E, Nixdorf B (2008) Praxistest zur Bewertung von Seen anhand des Phytoplanktons gemäß EU-WRRL. Endbericht zum LAWA-Projekt (O 5.05). In: Mischke U, Nixdorf B (Hrsg) Gewässerreport (Nr. 10): Bewertung von Seen mittels Phytoplankton zur Umsetzung der EU-Wasserrahmenrichtlinie, Eigenverlag der BTU Cottbus (BTUC-AR 2/2008, ISBN 978-3-940471-062). Cottbus, S 7-115

36. Wasserrahmenrichtlinie (EG-WRRL) Richtlinie 2000/60/EG des Europäischen Parlamentes und des Rates vom 23. Oktober 2000 zur Schaffung eines Ordnungsrahmens für Maßnahmen der Gemeinschaft im Bereich der Wasserpolitik. ABI. EG Nr. L 327/1, 22.12.2000

37. Oberflächengewässerverordnung (OGewV) Verordnung zum Schutz der Oberflächengewässer. Bundesgesetzblatt Jahrgang 2011 Teil I Nr. 37, ausgegeben zu Bonn am 25. Juli 2011

38. Wasserblick (2014) Wasserblick: Bund-Länder-Informations- und Kommunikationsplattform. http://www.wasserblick.net/servlet/is/42489. Zugegriffen: 16. März 2015

39. DIN EN ISO 6878:2004-09 (2004) Wasserbeschaffenheit - Bestimmung von Phosphor - Photometrisches Verfahren mittels Ammoniummolybdat (ISO 6878:2004)4

40. ISO 10260 (1992) Water quality - measurement of biochemical parameters - Spectrometric determination of the chlorophyll-a concentration.

41. Catherine A, Escoffier N, Belhocine A, Nasri AB, Hamlaoui S, Yéprémian C, Bernard C, Troussellier M (2012) On the use of the FluoroProbe ${ }^{\circledR}$, a phytoplankton quantification method based on fluorescence excitation spectra for large-scale surveys of lakes and reservoirs. Wat Res 46(6):1771-1784

42. DIN EN 15204:2006-12 (2006) Wasserbeschaffenheit - Anleitung für die Zählung von Phytoplankton mittels der Umkehrmikroskopie (UtermöhlTechnik)

43. Triantis T, Tsimeli K, Kaloudis T, Thanassoulias N, Lytras E, Hiskia A (2010) Development of an integrated laboratory system for the monitoring of cyanotoxins in surface and drinking waters. Toxicon 55(5):979-989

44. Humpage AR, Froscio SM, Lau H-M, Murphy D, Blackbeard J (2012) Evaluation of the abraxis strip test for microcystins ${ }^{T M}$ for use with wastewater effluent and reservoir water. Wat Res 46(5):15561565

45. ISO 20179 (2005) Water quality - determination of microcystins - method using solid phase extraction (SPE) and high performance liquid chromatography (HPLC) with ultraviolet (UV) detection.

46. Spoof L, Vesterkvist P, Lindholm T, Meriluoto J (2003) Screening for cyanobacterial hepatotoxins, microcystins and nodularin in environmental water samples by reversed-phase liquid chromatography- electrospray ionisation mass spectrometry. J Chromat A 1020(1):105-119

47. Oehrle SA, Southwell B, Westrick J (2010) Detection of various freshwater cyanobacterial toxins using ultra-performance liquid chromatography tandem mass spectrometry. Toxicon 55(5):965972
48. Furey A, Crowley J, Hamilton B, Lehane M, James KJ (2005) Strategies to avoid the mis-identification of anatoxin-a using mass spectrometry in the forensic investigation of acute neurotoxic poisoning. J Chromat A 1082:91-97

49. Lawrence JF, Niedzwiadek B, Menard C.(2005) Quantitative determination of paralytic shellfish poisoning toxins in shellfish using prechromatographic oxidation and liquid chromatography with fluorescence detection: collaborative study. J AOAC Int 88(6):1714-1732

50. Umweltbundesamt (2011) Entscheidungsunterstützungssytem für die Entwicklung einer systemspezifischen Strategie gegen das Vorkommen von Cyanotoxinen im Trinkwasser vom Einzugsgebiet bis zum Verbraucher. www.toxische-cyanobakterien.de. Zugegriffen: 17. März 2015 